\title{
Altas acumulaciones de precipitación en la península ibérica relacionadas con la borrasca Emma
}

https://doi.org/10.31978/639-19-010-0.451

\author{
Cecilia Marcos Martín¹ (cmarcosm@aemet.es) \\ Juanma Sancho Ávila² (jsanchoa@aemet.es)
}

${ }^{1}$ AEMET / Dirección de Producción e Infraestructuras / Área de Técnicas y Aplicaciones de Predicción ${ }^{2}$ AEMET / Dirección de Producción e Infraestructuras / Centro Nacional de Predicción

\begin{abstract}
RESUMEN
A finales de febrero una borrasca atlántica profunda, nombrada como Emma, desplaza una masa de aire subtropical húmeda y cálida que penetra por el suroeste de la Península y que interacciona con otra masa de aire polar continental fría y seca, procedente del norte de Europa, entrando esta por el noreste dirigida por un anticiclón situado en latitudes altas. La frontera entre ambas masas de aire se encuentra a primeras horas del día 28 sobre el cuadrante noreste y dicha interacción provoca nevadas en amplias zonas de la mitad norte peninsular.

En días posteriores se produce una activación de otras bajas atlánticas por el forzamiento que produce el desplazamiento hacia el sur de un embolsamiento de aire frío en niveles medios. Como consecuencia, una sucesión de frentes deja entre los días 26 de febrero y 5 de marzo altas acumulaciones de precipitación en amplias zonas de la Península, algunas de ellas en forma de nieve, llegando a superar los 200 litros acumulados en todo el periodo en algunos puntos.
\end{abstract}

PALABRAS CLAVE: Emma; borrasca atlántica; NAO; precipitación; nevadas; masa de aire.

\section{INTRODUCCIÓN}

El año 2018 comenzó con un índice NAO positivo que a finales de febrero tornó a negativo manteniéndose en estas condiciones hasta principios del mes de abril (véase figura 1). Índices NAO negativos (MET OfFICE, 2018) están asociados a anomalías positivas de precipitación en el sur y centro de Europa. Este episodio de NAO negativa ha estado asociado a la formación de una corriente en chorro zonal única en niveles altos de la troposfera a una latitud entre $30^{\circ}$ y $35^{\circ} \mathrm{N}$. Como consecuencia de ello la península ibérica se ha visto afectada por el paso continuo de un inusual número de borrascas atlánticas con sus frentes asociados que han dejado grandes cantidades de precipitación.

Figura 1.

Índice NAO observado enero-marzo 2018.

Fuente: NOAA.

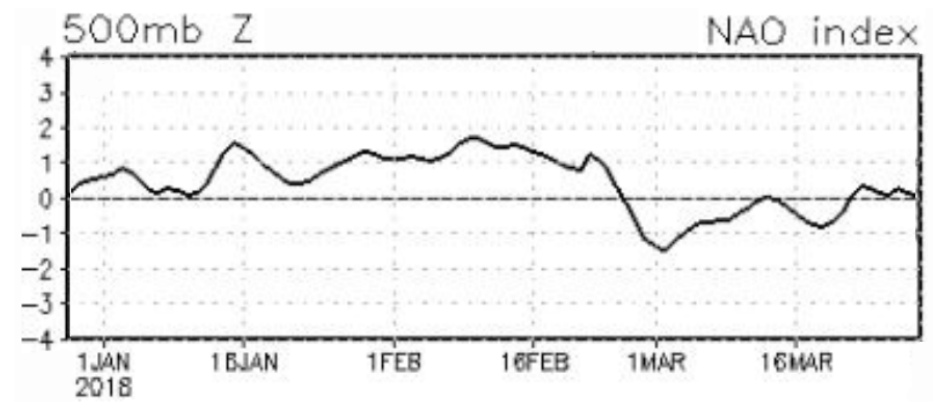


La primera de estas borrascas, nombrada como Emma, provoca un desplazamiento de una masa de aire subtropical húmeda y cálida haciendo que penetre por el suroeste de la Península. Al mismo tiempo, otra masa de aire polar continental frío y seco, procedente del norte de Europa, entra por el noreste de la Península dirigida por un anticiclón situado en latitudes altas. A primeras horas del día 28 de febrero la frontera entre ambas masas se encuentra sobre el cuadrante noreste provocando como consecuencia nevadas generalizadas en amplias zonas de la mitad norte peninsular que llegan a alcanzar cotas bajas.

Entre los días 26 de febrero y 5 de marzo el forzamiento debido a un desplazamiento hacia el sur de un embolsamiento de aire frío en niveles medios hace que se activen otras bajas atlánticas, prolongándose el episodio de tiempo adverso de forma generalizada tanto en la Península como en Canarias. Este episodio incluye nevadas, altas acumulaciones de precipitación, vientos fuertes y fenómenos adversos costeros.

Teniendo en cuenta la larga duración de este episodio y los diferentes efectos ocurridos a lo largo del mismo, este estudio se centra principalmente en los altos valores de precipitación recogidos los días 28 de febrero y 1 de marzo en la Península.

\section{SITUACIÓN SINÓPTICA}

El día 26 de febrero de 2018 se emite un aviso especial como consecuencia de la aproximación de una borrasca atlántica nombrada como Emma, que dará lugar a nevadas, lluvias intensas y vientos fuertes que afectaron los días posteriores a la Península y Canarias. Debido a esta situación se emitieron avisos naranjas por nevadas en amplias zonas del tercio norte peninsular, incluso en cotas muy bajas como la depresión del Ebro, que incluye Zaragoza y cotas bajas de Cataluña. También se emitieron avisos naranjas por rachas esperadas superiores a $95 \mathrm{~km} / \mathrm{h}$ en Canarias, y amarillos en gran parte de la Península, especialmente en la mitad sur.

Se pueden resaltar como características principales de Emma que llevaba asociados varios centros de vorticidad y núcleos fríos de hasta $-24{ }^{\circ} \mathrm{C}$ y un chorro del suroeste en niveles altos de hasta $100 \mathrm{kn}[(1852 / 3600) \mathrm{m} / \mathrm{s}]$ en algunos momentos, y que en la zona de difluencia llegó a superar los 125 kn sobre la Península. Esta borrasca, que en un principio se formó en el Atlántico y se mantuvo estacionaria varios días sobre las Azores, posteriormente se desplazó hacia el este debilitándose tras nueve días en su llegada a Europa.

a)

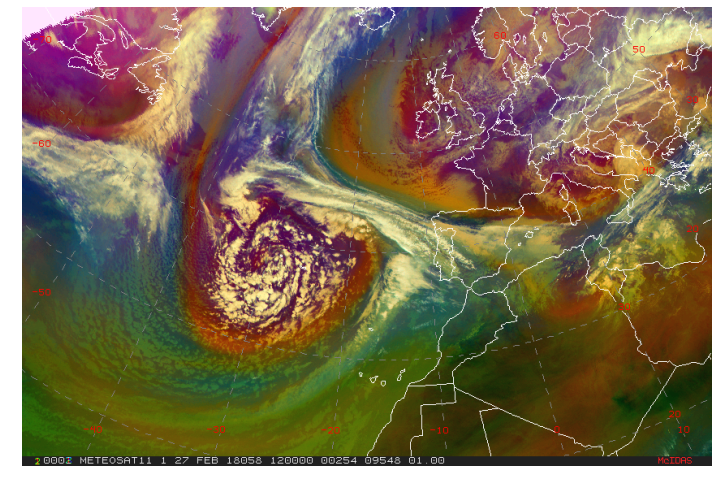

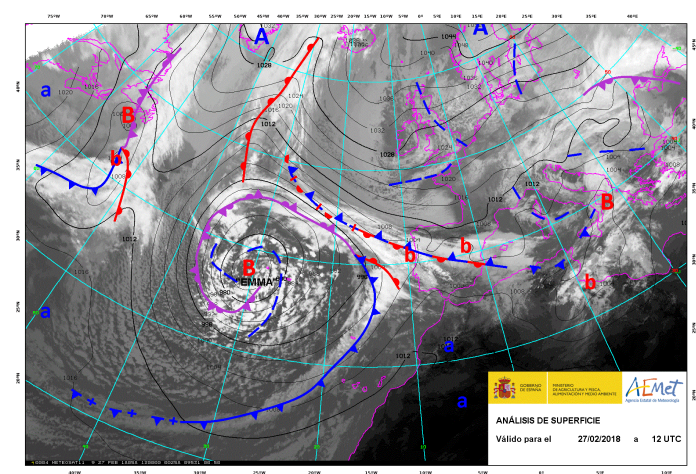

Figura 2. a) Imagen RGB de masas de aire y b) guía técnica de diagnóstico de niveles bajos sobre imagen del canal IR 10.8 para el 27 de febrero a las 12:00 UTC.

La situación sinóptica del día 27 de febrero a las 12:00 UTC está definida por la interacción de dos masas de aire con características muy diferentes cuya frontera se encuentra sobre el tercio norte peninsular, como se puede ver en la figura 2. Hay una masa polar fría y seca, impulsada por un flujo del nordeste debido a la alta de bloqueo situada sobre Islandia, que en días previos ya se encontraba sobre el cuadrante noreste de la Península. Al mismo tiempo una masa de aire tropical cálido y húmedo, cuyo avance está dirigido por el giro de la borrasca Emma, se desplaza hacia el norte, provocando un aumento del contraste térmico creando un frente estacionario sobre el tercio norte al tiempo que se desliza longitudinalmente una masa sobre la otra. 
a)

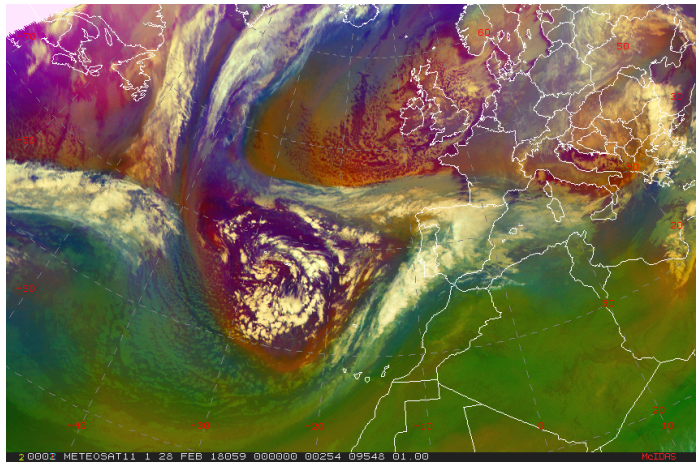

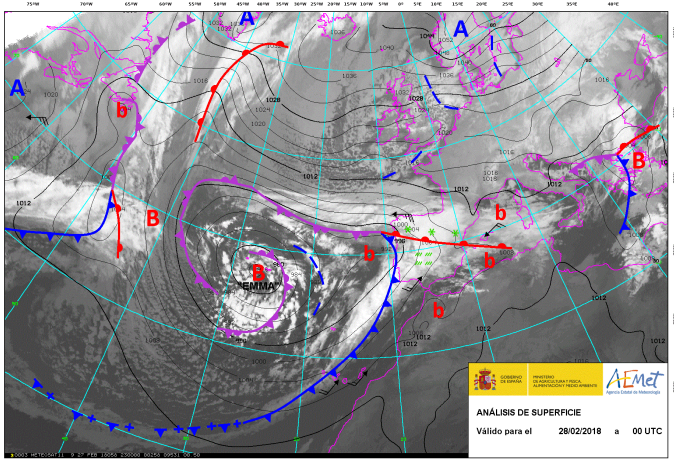

Figura 3. a) Imagen RGB de masas de aire y b) guía técnica de diagnóstico de niveles bajos sobre imagen del canal IR 10.8 para el 28 de febrero a las 00:00 UTC.

El 28 de febrero a las 00:00 UTC, el desplazamiento hacia el este de la borrasca Emma, que en días previos se encontraba estacionaria sobre el Atlántico, provoca que la masa cálida desplace hacia el norte a la masa fría, y que el frente estacionario evolucione a un frente cálido que deja nevadas generalizadas durante la madrugada del día 28 en gran parte de la mitad norte peninsular, alcanzando incluso cotas muy bajas (figura 3).

a)

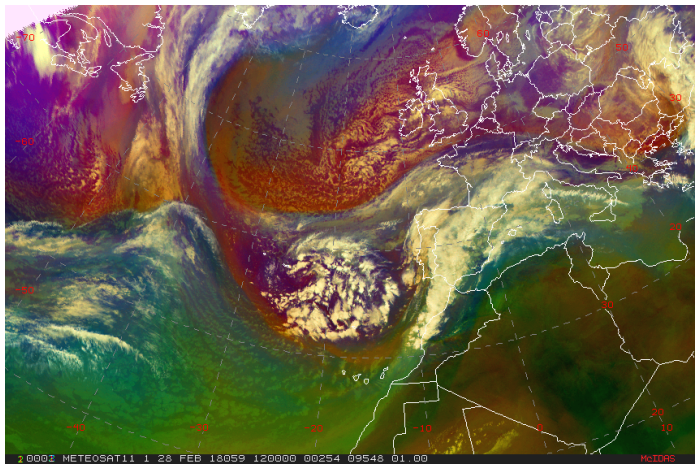

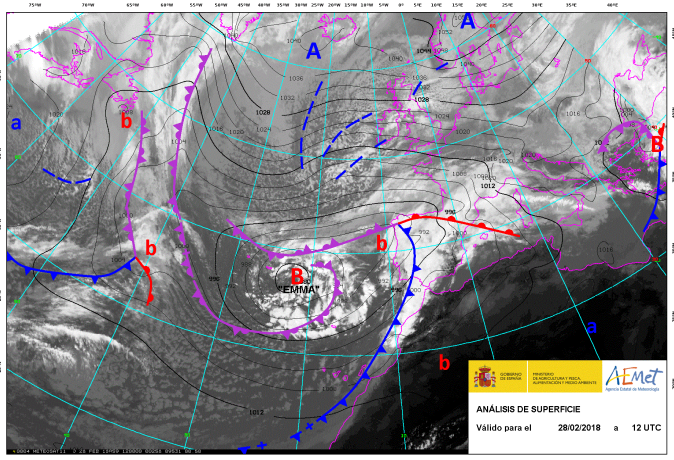

b)

Figura 4. a) Imagen RGB de masas de aire y b) guía técnica de diagnóstico de niveles bajos sobre imagen del canal IR 10.8 para el 28 de febrero a las 12:00 UTC.

El frente frío activo asociado a Emma (figura 4), posteriormente penetra por el suroeste provocando lluvias intensas y vientos fuertes en la mitad sur peninsular y en Canarias. La descarga fría postfrontal que alcanza el suroeste peninsular a primeras horas del 1 de marzo (figura 5), dio lugar a chubascos intensos en las mismas zonas, acompañados de alta actividad tormentosa y fuertes rachas de viento.

a)

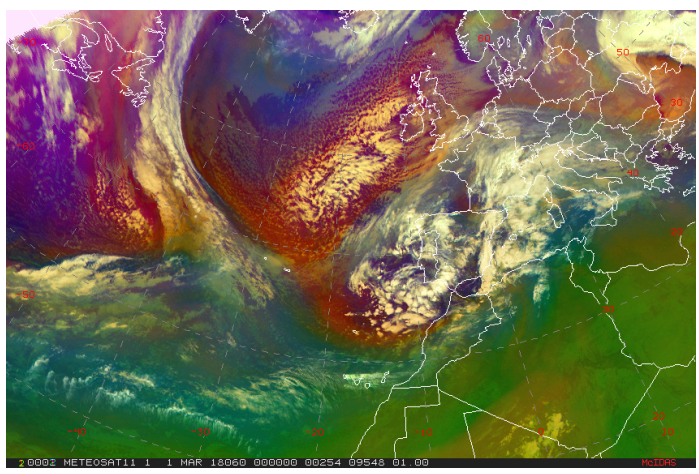

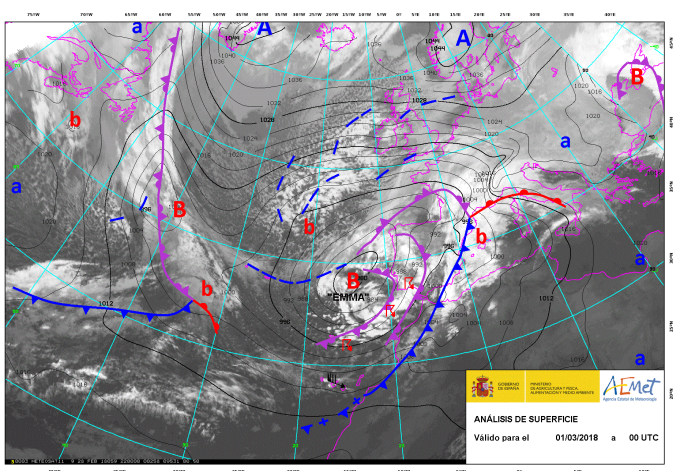

b)

Figura 5. a) Imagen RGB de masas de aire y b) guía técnica de diagnóstico de niveles bajos sobre imagen del canal IR 10.8 para el 1 de marzo a las 00:00 UTC. 


\section{PRINCIPALES IMPACTOS Y DATOS OBSERVADOS}

Las consecuencias del frente cálido que afecta al noreste peninsular en la madrugada del día 28, tal como se explica en el apartado de descripción de la situación sinóptica, son nevadas generalizadas en gran parte de la mitad norte peninsular. Aunque en general estas nevadas no produjeron altas acumulaciones de nieve, sí se alcanzaron espesores de $40 \mathrm{~mm}$ en zonas del Pirineo de Huesca y Lérida, así como en zonas del sistema Ibérico de Soria. La figura 7.a muestra el espesor en centímetros de nieve recogida el día 28 de febrero.

Especialmente reseñables fueron las nevadas caídas en la ciudad de León, con espesores de $11 \mathrm{~cm}$ de nieve, y Pamplona, con espesores de entre 10-15 cm, ambas con gran impacto en la población, ya que se produjeron en la madrugada afectando a los desplazamientos en horas punta. Según el reporte de SINOBAS (SIstema de Notificación de Observaciones Atmosféricas Singulares, https://sinobas.aemet.es/), esta fue una de las mayores nevadas registradas en Navarra durante los últimos 20 años. También hay que destacar que al producirse estas nevadas según avanzaba el frente cálido (figura 4), la capa de nieve se fundió con rapidez durante el día, disminuyendo rápidamente su impacto.

La figura 6 muestra sendas imágenes tomadas por webcams en las ciudades de Vitoria y Zaragoza el día 28 sobre las 12:00 horas locales.

a)

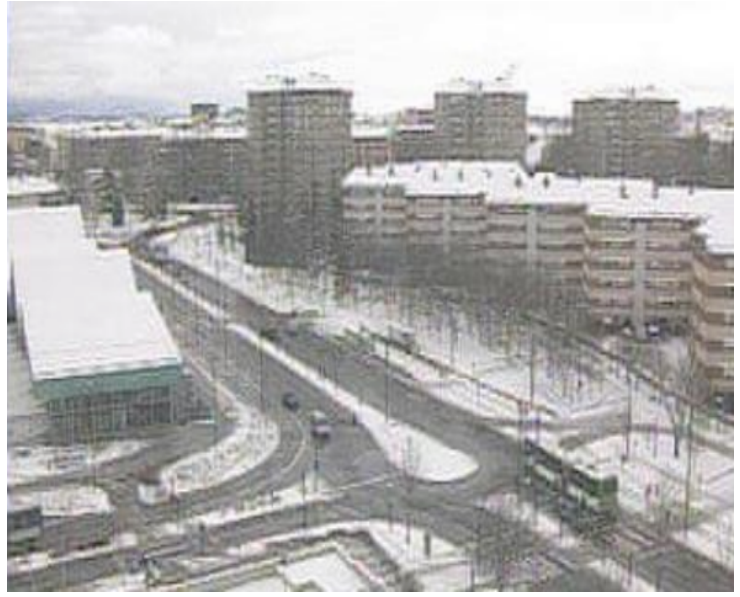

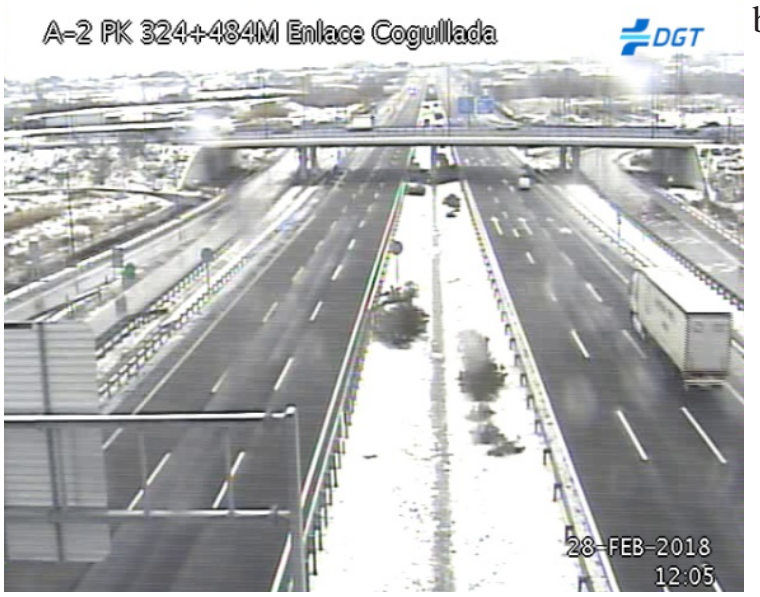

b)

Figura 6. Imágenes tomadas por webcams de las nevadas caídas la madrugada del 28 de febrero sobre: a) Vitoria y b) Zaragoza.

En la segunda mitad del 28 de febrero, el frente frío asociado a Emma penetra por el suroeste peninsular produciendo chubascos intensos, acompañados de actividad eléctrica y rachas muy fuertes en el cuadrante suroeste peninsular (figuras 7.b y 7.c). Destacables son las acumulaciones de precipitación recogidas de 170 mm en Puerto del Pico (Ávila) y de más de 120 mm en zonas de Extremadura y Andalucía occidental. Además, este día se alcanza una efemérides de precipitación máxima diaria para los meses de febrero de 34,2 mm en la estación de Colmenar Viejo.

Debido al avance del frente frío, el día 1 de marzo, las rachas muy fuertes se extendieron a amplias zonas del interior peninsular, llegando a superar los $100 \mathrm{~km} / \mathrm{h}$ en zonas altas (figura 8.b). En concreto, en la estación de Madrid-Retiro se alcanza este día una efemérides de $84 \mathrm{~km} / \mathrm{h}$ para un mes de marzo. La descarga fría postfrontal tras el paso del frente frío dejó nuevamente chubascos generalizados en la mitad oeste peninsular (figura 8.a), extendiéndose las tormentas a lo largo del valle del Guadalquivir y el litoral sur, así como a zonas de la meseta sur (figura 8.c).

Como consecuencia de este temporal de nieve, lluvia y viento, cuatro personas perdieron la vida, se cerraron más de 300 kilómetros de carreteras en 12 provincias y miles de alumnos no pudieron asistir a sus clases en las comunidades del País Vasco, Aragón, La Rioja, Asturias, Comunidad Valenciana y Canarias. 

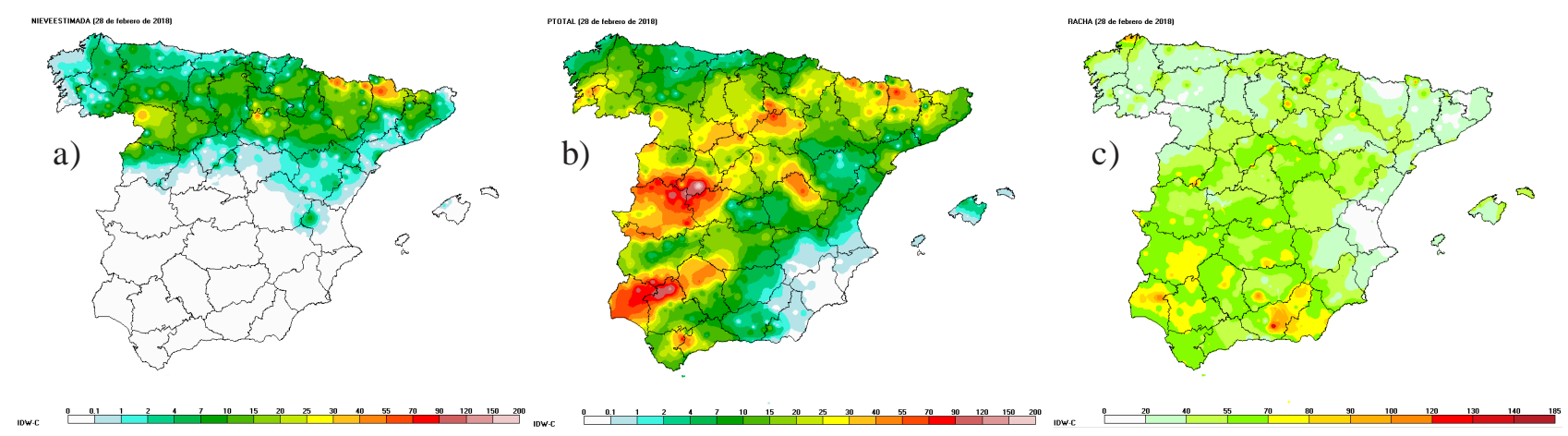

Figura 7. a) Nieve estimada, b) precipitación total y c) racha máxima para las 24 horas del 28 febrero obtenidas a partir de la base de datos climatológica de AEMET.
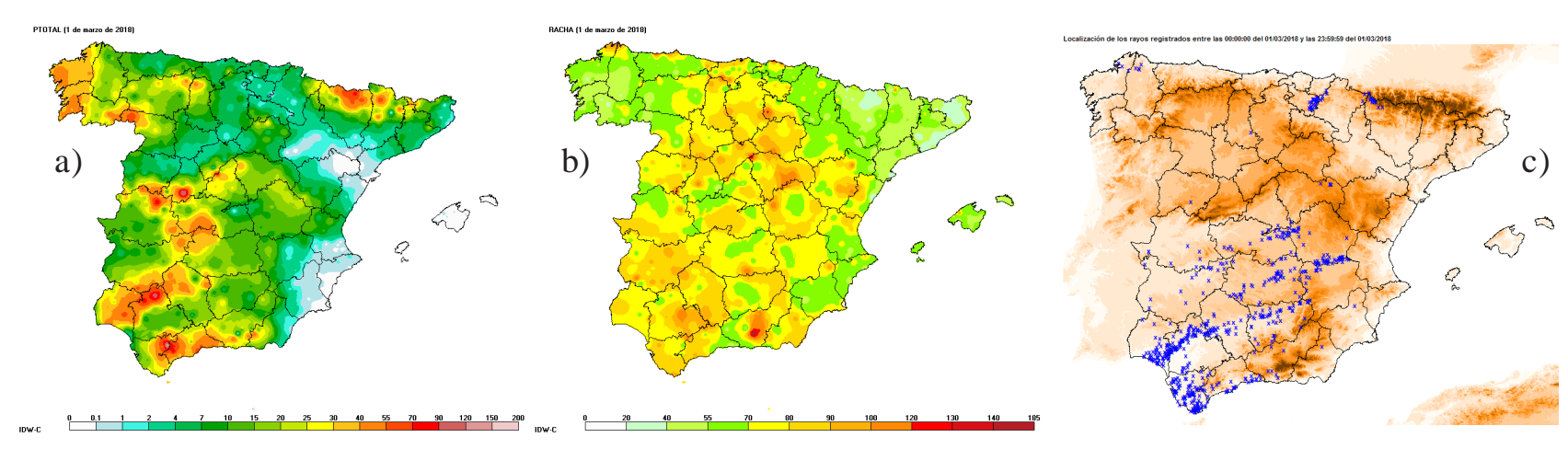

Figura 8. a) Precipitación total, b) racha máxima y c) número de rayos para las 24 horas del 1 de marzo obtenidas a partir de la base de datos climatológica de AEMET.

\section{PRECIPITACIÓN PREVISTA POR EL MODELO DEL ECMWF}

\subsection{Nevadas en el tercio norte peninsular}

Como se ha mencionado en la introducción, este estudio se centra principalmente en los altos valores de precipitación recogidos los días 28 de febrero y 1 de marzo en la Península.

Para este estudio utilizamos la pasada de las $00 \mathrm{~h}$ del día 27 de febrero. En primer lugar nos fijamos en los campos de precipitación del modelo determinista HRES-IFS. En la figura 9 vemos los campos de previsión de nieve acumulada en 12 horas válidos para el 28 de febrero a las 12:00 h (figura 9.a) y el 1 de marzo a las 00:00 h (figura 9.b). Estos campos muestran que las nevadas se iban a concentrar principalmente durante la primera mitad del día 28 de febrero, manteniéndose durante el resto de la jornada en zonas montañosas del Cantábrico, sistema Ibérico y Pirineos.

Atendiendo a un enfoque más probabilista, en la figura 10 se pueden ver los campos de probabilidad de nevada acumulada durante las 24 horas del día 28 , superior a tres umbrales $(5,10$ y $20 \mathrm{~mm}$ de agua equivalente) según el ENS-IFS. Según el campo con umbral $5 \mathrm{~mm}$, las zonas de nevada son muy similares a las dadas por el modelo determinista (figura 9). En este caso podemos ver que el ENS-IFS aporta un claro valor añadido al modelo determinista ya que, tal como vemos en los campos con umbrales 10 (figura 10.b) y 20 (figura 10.c) mm, este modelo probabilístico señala zonas tales como León, Pamplona y la Ibérica de Soria, donde se registraron acumulaciones de nieve por encima de $10 \mathrm{~cm}$, que no destacaban claramente en el modelo determinista. Esto demuestra la utilidad del modelo probabilístico en el corto plazo. 
a)

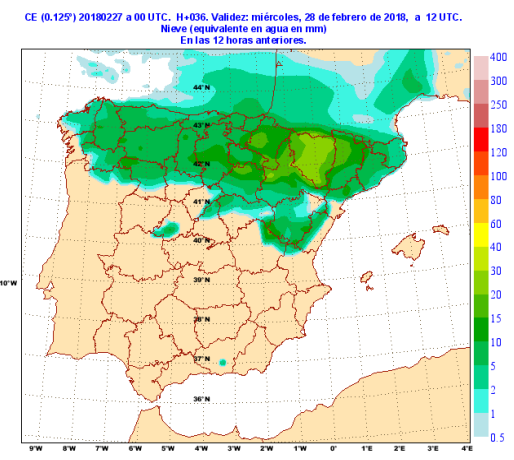

b)

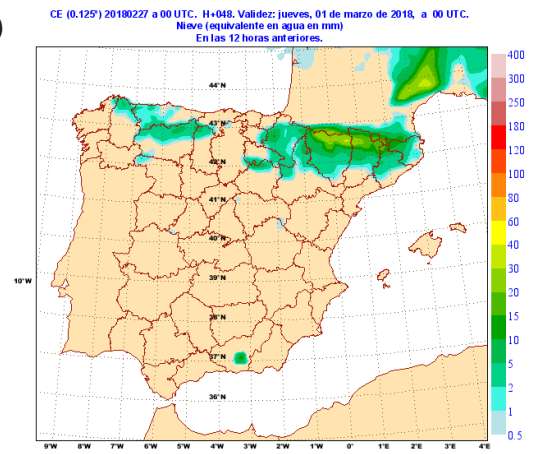

Figura 9. Campos de predicción de nieve acumulada a) en las 12 primeras y b) las 12 últimas horas del día 28 de febrero (pasada del 27 de febrero a las $00 \mathrm{~h}$ ).

a)

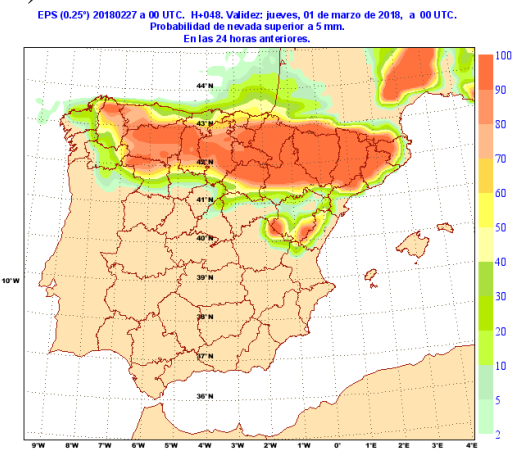

b)

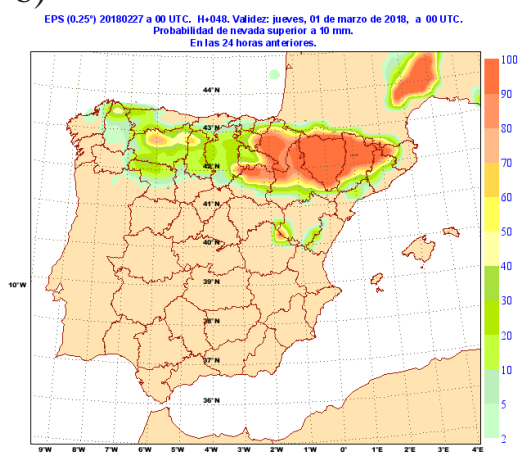

c)

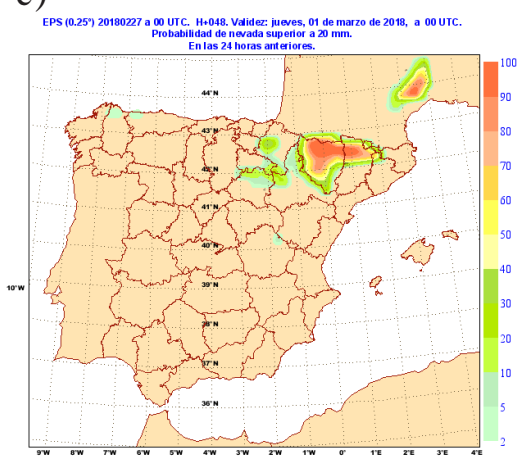

Figura 10. Probabilidad de nevada superior a los umbrales de a) $5 \mathrm{~mm}$, b) $10 \mathrm{~mm}$ y c) $20 \mathrm{~mm}$ acumulada en las 24 horas del día 28 de febrero (pasada del 27 de febrero a las 00 h).

Un índice muy útil a la hora de detectar fenómenos inusuales o extremos, que no necesariamente severos, es el EFI (Extreme Forecast Index) (OwEns y Hewson, 2018 - EFI). Este índice se calcula comparando la función de distribución de probabilidad obtenida con el ENS-IFS y la correspondiente distribución del $M$-climate o climatología del modelo ${ }^{1}$. Para obtener el EFI se hace un cálculo ponderado (dando más peso a los valores extremos del $M$-climate) del área comprendida entre la función de distribución del probabilidad del ENS y la correspondiente al $M$-climate, sin considerar los miembros que están fuera de los límites del M-climate. Para considerar el efecto de estos últimos existe otro índice llamado SOT (Shift of Tails) (OwENS y Hewson, 2018 - SOT).

Los valores del EFI están comprendidos entre - 1 y 1 . En cuanto a su interpretación (en valor absoluto), valores entre 0,5 y 0,8 indicarían un evento inusual. Valores por encima de 0,8 , indicarían un evento muy inusual o extremo, significando el valor 1 que todos los miembros del ENS superan los límites del M-climate.

La figura 11 muestra el campo correspondiente al EFI para precipitación en forma de nieve acumulada durante las 24 horas del día 28. Este campo indicaba valores por encima de 0,7 en amplias zonas del tercio norte, con valores especialmente altos, mayores que 0,8 , en el valle del Ebro y zona norte de Castilla y León. Es destacable que el EFI en Pirineos no tiene una señal importante ya que las nevadas en esa zona en estas fechas no son algo inusual. Sin embargo, en el valle del Ebro, la señal EFI indica un fenómeno extremo ya que apuntaba a nevadas en cotas muy bajas que no son habituales.

\footnotetext{
La climatología del modelo (M-climate) se calcula para cada alcance y tiene en cuenta cinco semanas centradas en el día de la pasada sobre la que se quiere hacer el cálculo. Dentro de estas cinco semanas se cogen 9 pasadas, siempre de las 00, siendo la central la correspondiente al lunes o jueves anterior más próximo a la pasada de interés, y los cuatro lunes/jueves anteriores y posteriores. Se reprocesa un grupo de 11 miembros para cada pasada y para ese mismo día de los últimos 20 años, lo que hace un total de $20 \times 9 \times 11=1980$ valores de predicción reprocesados para calcular la climatología.
} 


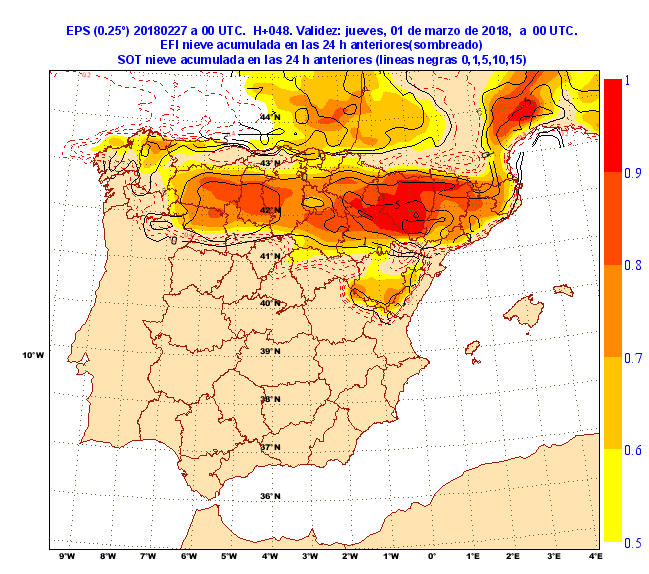

Figura 11. EFI de nieve acumulada en las 24 horas del día 28 de febrero (pasada del 27 de febrero a las $00 \mathrm{~h}$ ).

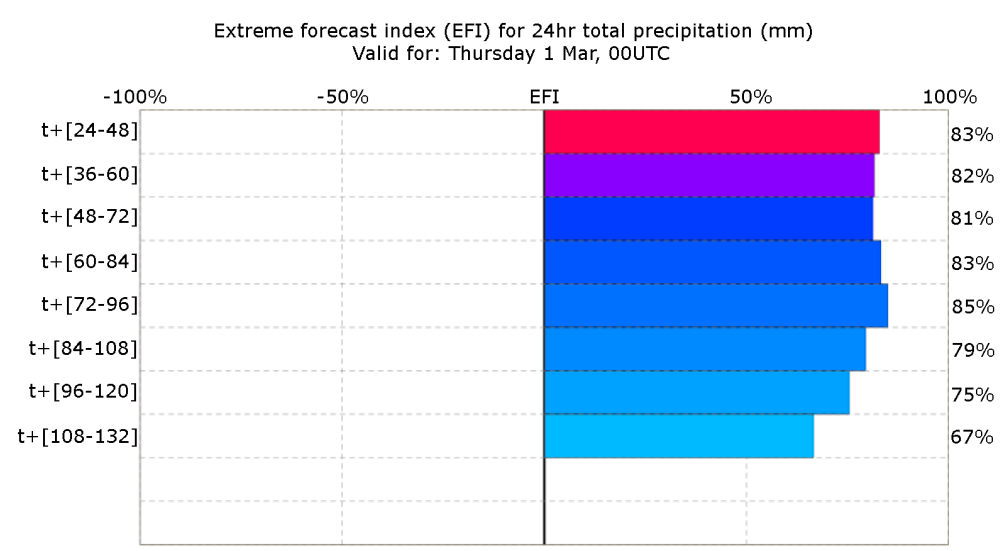

Figura 12. Valores de EFI para Zaragoza válidos para las 24 horas del día 28 de febrero.

La figura 12 muestra la evolución del EFI de precipitación total en 24 horas para Zaragoza en las últimas pasadas (cada 12 horas), estando situadas por orden de antigüedad, siendo las más recientes las situadas en la parte superior. En este caso los valores del EFI se ajustan a una escala porcentual, representando un $100 \%$ un EFI igual a 1 (parte derecha de la gráfica). Este tipo de gráfica es útil porque muestra si hay consistencia en la señal EFI en las últimas pasadas. En este caso, la gráfica muestra 8 pasadas. Todas ellas dan señales altas avisando, con al menos cinco días de antelación, de una precipitación inusualmente alta. Siendo todas ellas bastante consistentes, según se van aproximando al día de validez, el EFI se va ajustando, tomando valores ligeramente mayores en las últimas pasadas, superando el $80 \%$ que indica un fenómeno extremo. Este es un ejemplo de consistencia entre pasadas que muestra una alta predecibilidad de la atmósfera es este caso y, por lo tanto, una mayor fiabilidad en la predicción.

La figura 13 muestra las gráficas CDF (Cumulative Distribution Function o función de distribución acumulativa) (OwEns y Hewson, 2018 - CDF) para el mismo día y lugar (Zaragoza). En esta gráfica, disponible a través de eccharts (https://www.ecmwf.int/en/forecasts/eccharts), se representan en el eje de abscisas los valores de precipitación y en el de ordenadas la probabilidad de que los valores previstos sean inferiores a dichos valores de precipitación. Las gráficas en colores corresponden a las funciones de distribución acumulativas de las últimas pasadas del ENS-IFS, manteniéndose el mismo orden de los colores de la figura 12. La línea negra corresponde a la función de distribución acumulativa del $M$-climate. Una mayor pendiente en estas funciones indica una mayor confianza o convergencia de un mayor número de miembros a un intervalo de valores de precipitación más pequeño, indicando una mayor fiabilidad en la predicción del ENS. Por otro lado, cuanto más alejadas de la distribución del $M$-climate están las funciones del ENS, más inusual o extremo es el evento que predicen. Y cuanto más a la derecha, más severo. Cuando las curvas se aproximan unas a otras en las últimas pasadas, es señal de que hay una convergencia en las predicciones, y por lo tanto, una mayor fiabilidad de la predicción.

En este caso se puede ver cómo según avanzan las pasadas en el tiempo, el ENS apunta a acumulaciones mayores de precipitación en 24 horas, proporcionando los valores más altos, la última pasada. Además, estas últimas pasadas se alejan más de la función correspondiente al $M$-climate, indicando un fenómeno más inusual. Es importante notar que la pendiente de todas las gráficas, aunque parecida, no es muy elevada, indicando una dispersión alta de los miembros, y por lo tanto, denotando un evento de baja predecibilidad.

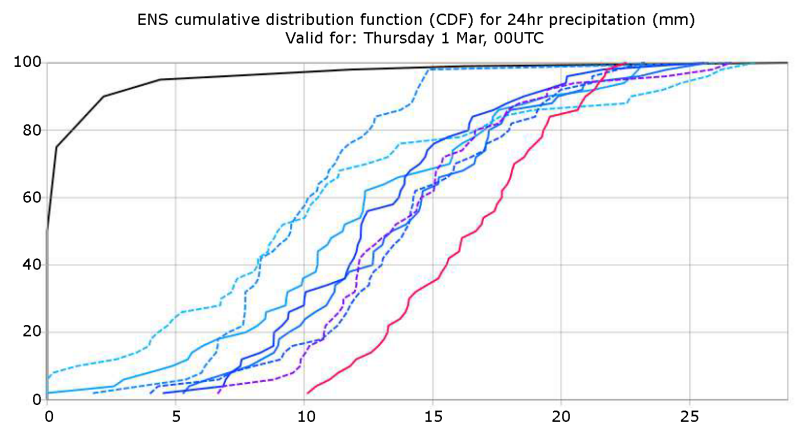

Figura 13. Valores de CDF de acumulación de precipitación total en 24 horas para Zaragoza válida para las 24 horas del día 28 de febrero. 
Teniendo en cuenta que los valores observados en el valle del Ebro en el entorno de la ciudad de Zaragoza (punto al que se refiere la gráfica) estaban en torno a los 10-15 cm de nieve, se puede decir que la última pasada del ENS sobreestima las cantidades de precipitación caídas, ajustándose mucho más las anteriores pasadas que entre sí se mantenían más estables y daban valores más cercanos a lo que realmente ocurrió. Esto demuestra por una parte que la última pasada del modelo no tiene por qué ser la mejor y, por otra, el valor que tiene que estudiar la historia del modelo y su comportamiento para cada situación.

\subsection{Altas acumulaciones de precipitación asociadas al frente frío}

Durante el día 28, el frente frío asociado a Emma entró por el suroeste peninsular dejando altas acumulaciones de precipitación en la mitad oeste. Estas precipitaciones se prolongaron gracias a la descarga fría postfrontal durante el día 1 de marzo.

Las figuras 14.a y 14.b muestran los campos del HRES-IFS de acumulación de precipitación en 24 horas para los días 28 de febrero y 1 de marzo respectivamente. Se puede ver como los chubascos previstos para el día 28 de febrero son generalizados en casi toda la Península y los previstos para el 1 de marzo, aunque también afectan a la mayor parte del territorio peninsular, se centran sobre todo en sistemas montañosos.

a)

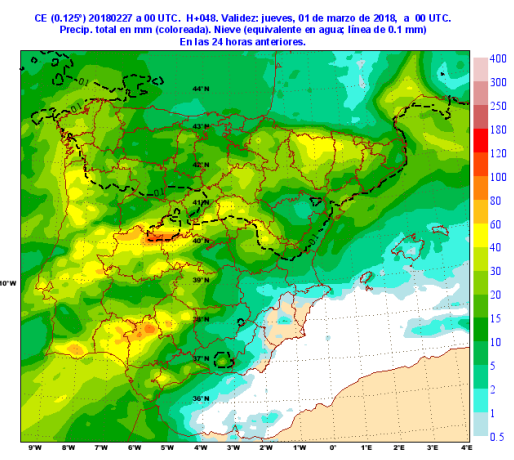

b)

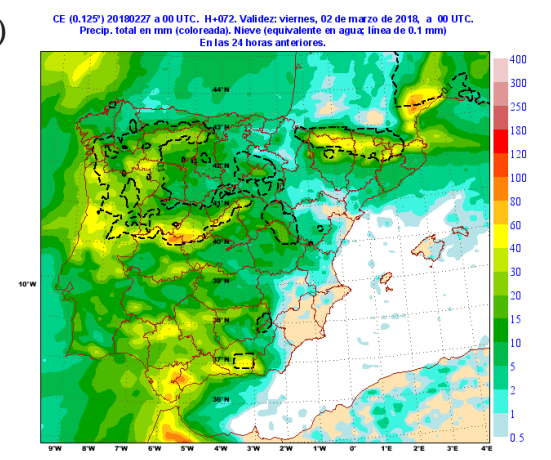

Figura 14. Campos de predicción de acumulación de precipitación total correspondientes al día a) 28 de febrero y b) 1 de marzo (pasada del 27 de febrero a las $00 \mathrm{~h}$ ).

Los campos de EFI de precipitación total acumulada en 24 horas para los días 28 de febrero y 1 de marzo que se muestran en las figuras 15 .a y 15 .b respectivamente, muestran valores superiores a 0,8 , que indican una situación muy inusual, exceptuando el extremo norte y el sureste peninsular.

a)

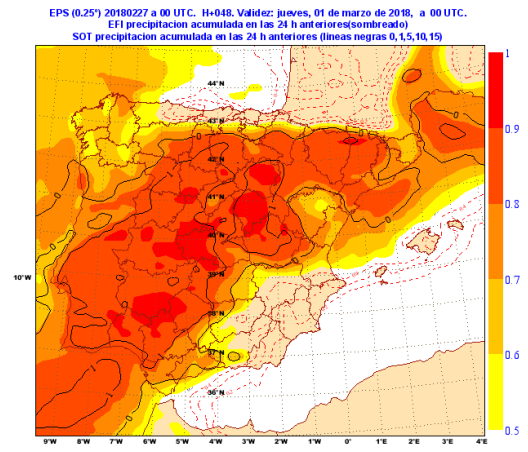

b)

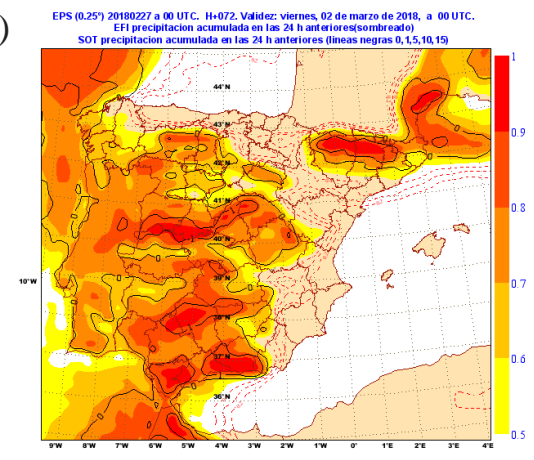

Figura 15. EFI de precipitación total acumulada en las 24 horas del día a) 28 de febrero y b) 1 de marzo (pasada del 27 de febrero a las $00 \mathrm{~h}$ ).

Como ejemplo se muestran la evolución de los EFI y las CDF en Cazalla de la Sierra, localidad situada en la sierra norte de Sevilla (véanse los mapas de la figura 16) donde se alcanzaron acumulaciones de precipitación en 24 horas de 126,2 mm el día 28 de febrero y 99,4 mm el 1 de marzo. La mayor acumulación de precipitación se recogió entre las 12 h del día 28 y las 12 h del día 1, con 168,9 mm. 

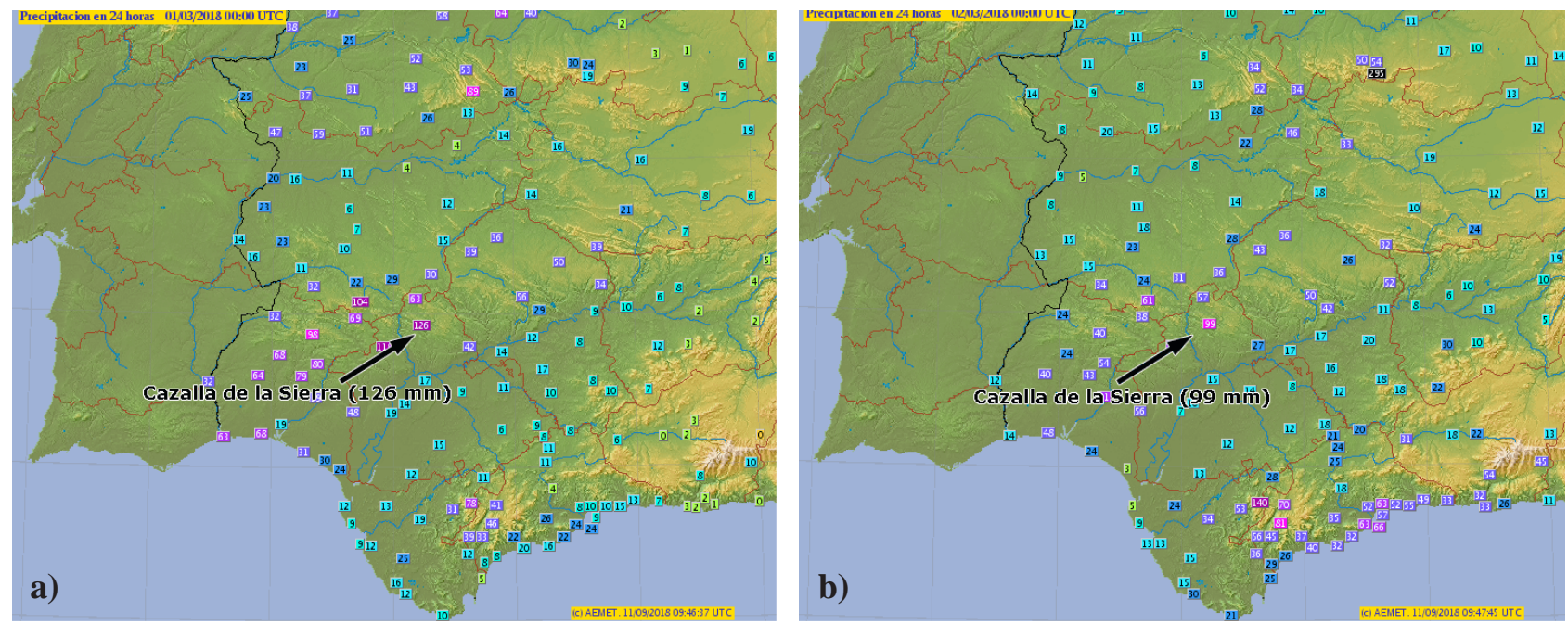

Figura 16. Mapa de acumulaciones de precipitación recogidas en las 24 horas del a) 28 de febrero y b) 1 de marzo.

Las figuras 17 y 18 muestran la evolución de los EFI y las CDF del ENS en las últimas pasadas para el día 28 de febrero en Cazalla de la Sierra. Es reseñable ver que con cuatro días de antelación las señales de EFI ya estaban por encima de 0,8 , manteniéndose aproximadamente estables hasta la última pasada, indicando un fenómeno muy inusual o extremo. En la gráfica de las CDF vemos que, según la climatología del modelo para esa zona, las acumulaciones de precipitación en esta época son muy bajas, con una alta probabilidad de que estén por debajo de $10 \mathrm{~mm}$. En este caso todas las pasadas se alejan de manera considerable hacia la derecha de la climatología del modelo, indicando precipitaciones previstas excepcionalmente anormales. Según se aproximan al momento de validez se puede apreciar una tendencia de aumento de la adversidad con cada pasada, siendo la última (D+1) la que más precipitación pronostica, estando finalmente en este caso, más acorde con la observación. Además, esta última pasada también aumenta sensiblemente la pendiente de la CDF, y por tanto la fiabilidad.

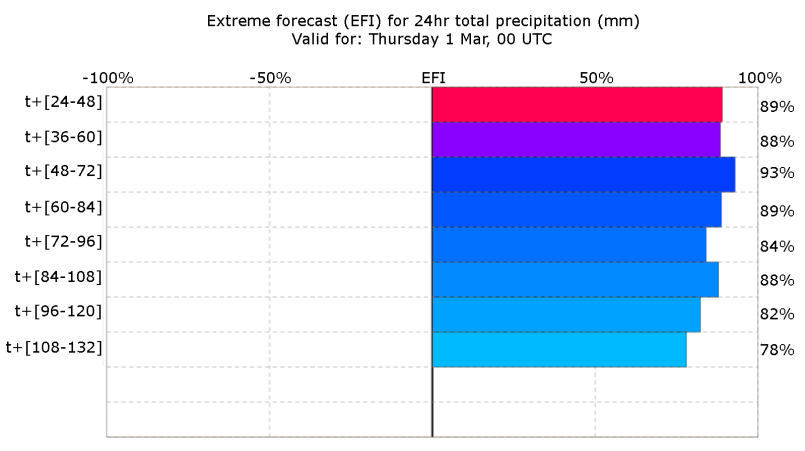

Figura 17. Valores de EFI de precipitación total acumulada en las 24 horas del día 28 de febrero en Cazalla de la Sierra.

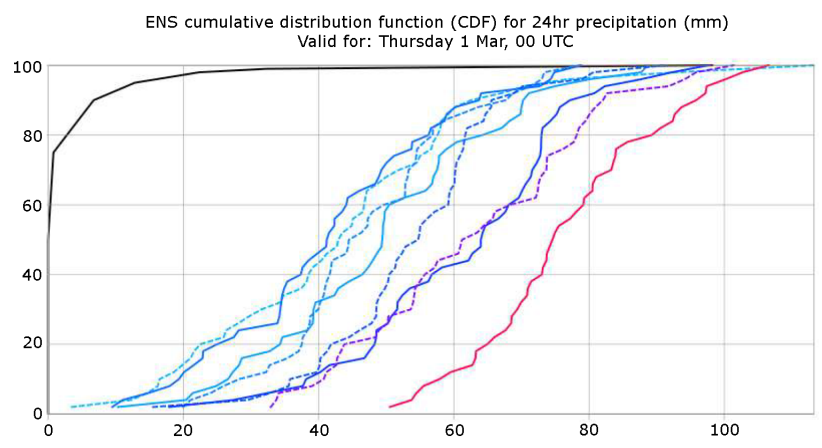

Figura 18. Valores de CDF de precipitación total acumulada en las 24 horas del día 28 de febrero en Cazalla de la Sierra.

Si nos fijamos en el comportamiento de los EFI y CDF para el día 1 de marzo en Cazalla de la Sierra (figuras 19y 20), vemos que los EFI se mantienen con valores inusualmente altos y las CDF, aunque pronostican valores altos de precipitación, dichos valores son menores que los previstos para el día anterior, tal como ocurrió realmente. También es destacable la convergencia de las curvas en las últimas pasadas y el aumento de la pendiente, indicando una disminución de la dispersión entre los miembros del ENS, en la última pasada. 


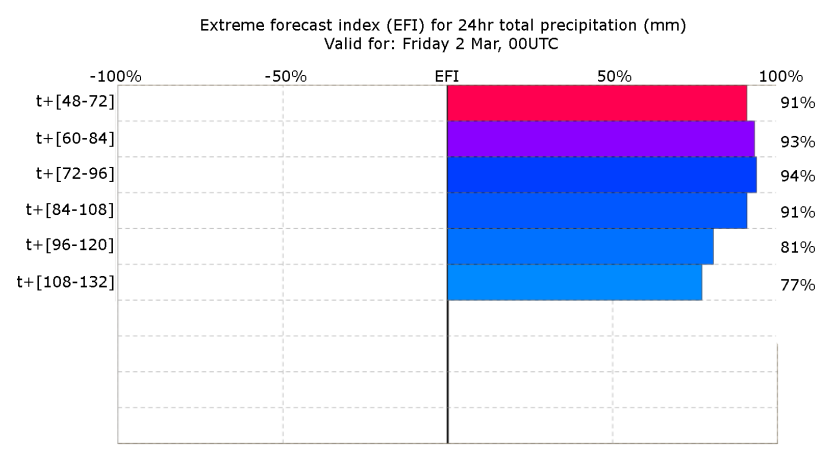

Figura 19. Valores de EFI de precipitación total acumulada en las 24 horas del día 1 de marzo en Cazalla de la Sierra.

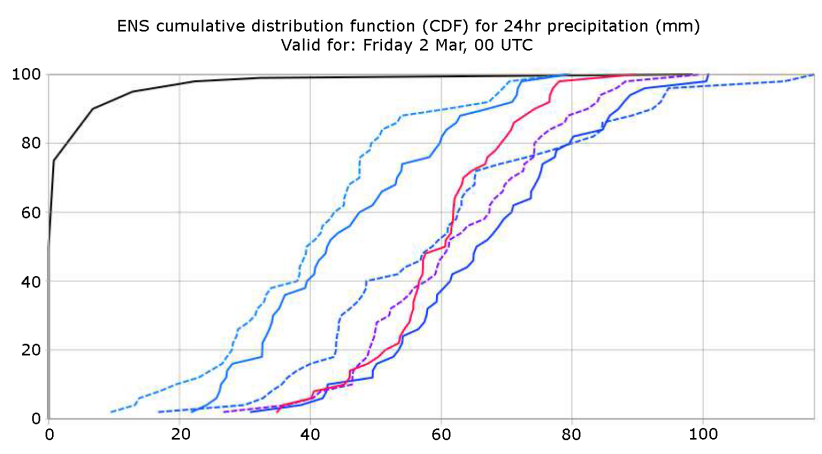

Figura 20. Valores de CDF de precipitación total acumulada en las 24 horas del día 1 de marzo en Cazalla de la Sierra.

\section{CONCLUSIONES}

Debido a la situación de NAO negativa que se ha dado este invierno, se han sucedido numerosas borrascas atlánticas en el país que han provocado efectos adversos tanto en la Península como en Canarias. Una de las más activas ha sido la nombrada como Emma, que dejó un temporal de nieve, lluvia y viento, en los últimos días de febrero y primeros de marzo.

En este trabajo se muestra la utilidad de herramientas probabilísticas tales como los campos de EFI y funciones CDF, así como la evolución de las mismas entre las últimas pasadas del modelo. También se ofrece una orientación sobre la interpretación de estos productos y su funcionamiento.

Se ha visto que una visión de la evolución de los resultados probabilísticos del modelo ofrece una gran cantidad de información útil, concluyendo que la última pasada no tiene por qué ser la más fiable.

\section{REFERENCIAS}

Met OfFICE, 2018. NorthAtlantic Oscillation: https://www.metoffice.gov.uk/learning/atmosphere/north-atlanticoscillation.

Owens, R. G., y Hewson, T.D., 2018. Extreme Forecast Index: https://confluence.ecmwf.int/display/FUG/ Extreme+Forecast+Index+-+EFI.

Owens, R. G., y Hewson, T. D., 2018. Shift of Tails: https://confluence.ecmwf.int/display/FUG/ Calculating+the+Shift+of+Tails+-+SOT.

Owens, R.G., y Hewson, T.D., 2018. Cumulative Distribution Function: https://confluence.ecmwf.int/display/ FUG/Cumulative+Distribution+Function\%2C+Probability+Density+Function . 